\title{
Frequency-dependent strategy selection in a hunting game with a finite population
}

\author{
Shuai Zhang, ${ }^{\mathrm{a}, *}$, Ruaridh Clark ${ }^{\mathrm{b}}$, Yunke Huang ${ }^{\mathrm{a}}$ \\ ${ }^{a}$ School of Marine Science and Technology, Northwestern Polytechnical University, Xi'an, \\ Shaanxi, P. R. China \\ ${ }^{b}$ Department of Mechanical and Aerospace Engineering, University of Strathclyde, Glasgow, \\ $U K$
}

\begin{abstract}
This paper considers a hunting game in the "playing the field" model, in which an individual within a group has to choose from two survival strategies: the group hunting strategy or the individual hunting strategy. The group hunting strategy aims at hunting more dangerous, larger prey, that are far beyond a single individual's capture ability, where the return is greater but the risk is higher. While the individual hunting strategy aims at hunting small prey that can be easily captured by an independent individual, where the return is less but the risk is lower. Evolutionary game theory is used to investigate the selection dynamics of a two-strategy game with a finite population. This reveals the existences of the stable/unstable equilibrium points and evolutionarily stable strategies when there is the frequency-dependent strategy selection in the hunting game. The evolutionarily stable state is found to be not always unique because the system of the hunting game can have multiple equilibrium points. It is shown that a stable equilibrium point will always act as an evolutionarily stable strategy, while an unstable equilibrium point cannot resist invasion from a mutation. The population fitness cannot always reach the optimum level when applying the evolutionary process with the fitness difference function.
\end{abstract}

Keywords: frequency-dependent selection, evolutionary game theory, playing

${ }^{*}$ Corresponding author at: School of Marine Science and Technology, Northwestern Polytechnical University, China

Email address: shuaizhang@mail.nwpu.edu.cn (Shuai Zhang)

Preprint submitted to Journal of ${ }^{A} T_{E} X$ Templates

January 10, 2020 
the field, hunting game

\section{Introduction}

Understanding selection dynamics is a fundamental problem in evolutionary biology [1]. Evolutionary game theory $[2,3]$ has become a powerful framework to study the two-player, two-strategy game, including the Hawk-Dove game (also known as Snowdrift or Chicken game) [4, 5], which describes competition between aggressive and passive behavioral strategies, and the Prisoner's Dilemma game [6], which describes competition between cooperative and defective behavioral strategies. These games represent different social dilemmas and have been extensively studied for understanding the evolution of cooperation from the weighted complex networks $[7,8,9]$. The payoff matrix for a game with two strategies $\mathrm{A}$ and $\mathrm{B}$ is

$$
\begin{aligned}
& \text { A } B \\
& \begin{array}{l}
A \\
B
\end{array}\left(\begin{array}{ll}
a & b \\
c & d
\end{array}\right)
\end{aligned}
$$

The concept of an evolutionarily stable strategy (ESS) $[2,3,10]$ is widely used to analyze the equilibrium states in evolutionary games. An ESS is a strategy which, if the majority of individuals adopt it, then there is no "mutant"

5 strategy that could achieve a higher fitness and hence it resists invasion by a rare mutation. Most prior work has assumed that the population is infinite and the individuals are randomly matched, in pairs, to play a symmetric two-player game $[11,12,13,14,15,16,17,18]$. This "pairwise random matching" model has four selection scenarios, describing evolutionary game dynamics among two strategies $[10,12]$.

(1) A-dominant. If $a>c$ and $b>d$, then $\mathrm{A}$ is a strict Nash equilibrium, and therefore an ESS.

(2) Bi-stable. If $a>c$ and $b<d$, the equilibrium point in the interior where $x_{A}=\frac{d-b}{a+d-b-c}$ is unstable $\left(x_{A}\right.$ is the frequency of individuals playing 
strategy A), and there are two absorbing states: the pure strategy A and the pure strategy B. Both A and B are strict Nash equilibria.

(3) Polymorphic. If $a<c$ and $b>d$, the equilibrium point in the interior where $x_{A}=\frac{d-b}{a+d-b-c}$ is stable. Neither A nor B is a Nash equilibrium. A mixed strategy is an ESS.

(4) Neutral case. If $a=c$ and $b=d$, the two strategies are equally good and the selection dynamics are inconsequential.

The classical Prisoner's Dilemma game and the Hawk-Dove game are both inadequate for describing social interactions between animals. One key issue is that both of these games assume that the individuals are randomly matched, in pairs, or play a symmetric two-player game in each round. It is worth noting that the individuals may also regard nature or environment as a potential opponent [19], in which an individual's expected rate of reproduction, or fitness, is not determined by another player's strategy, but jointly determined by its own strategy and what other strategies are present in the population and on their frequencies $[2,3,20]$. In this case, it is no longer a two-player game, but a multi-player game with the playing the field model designed for such applications [3, 21]. It generalizes the "pairwise random matching model" by assuming the individual's fitness depends linearly on the population or the strategy frequency, allowing the analysis of a wide range of situations where individuals 35 interact simultaneously in groups larger than pairs [20, 22, 23].

A typical playing the field problem is seen in the "tragedy of commons", in which there are always too many free riders, who do not contribute but enjoy the benefits of other members' contributions [24]. This issue is widely studied by the public goods game where each member's benefit is jointly determined 40 by the whole group [25, 26, 27, 28]. For example, the strike is such a "playing the field" problem, where the opponent of a worker is the employer or the environment rather than other workmates. However, the worker's payoff is jointly determined by its own strategy and the frequency distribution of other individuals' strategies. Other examples can be observed in sex ratios [29], habitat 45 distribution in birds [30] and stock market [31]. 
Another issue with the classical two-player games is that the payoff matrix of the classical two-player game is a constant matrix, as shown in Eq. (1). The constant model is not suitable for the playing the field problem, as the payoff or fitness may not be constant if it is population or frequency dependent.

In this paper, we introduce a two-strategy game with finite players in playing the field model which is termed as the hunting game, in which an individual in a group has to choose a survival strategy. The choice is between the group hunting strategy and the individual hunting strategy, with the individual's payoff jointly determined by its own strategy and the frequency distribution of other individuals' strategies. We focus our attention on the evolutionary dynamics, the stable/unstable equilibrium points and the Evolutionarily Stable Strategies (ESSs) in the hunting game with frequency-dependent strategy selection.

The paper is organized as follows. In Section 2, the paper opens with a simple strike example, which presents a trivial case where the individual's payoff is 60 jointly determined by its own strategy and the frequency distribution of other individuals' strategies. Section 3 then provides a general two-strategy game in the playing the field model, in which the selection dynamics of the game are formulated as a Moran process with frequency-dependent fitness. Section 4 provides the formulation of the hunting game, where two specific types of hunting game are analyzed in terms of strategy selection dynamics, stable/unstable equilibrium points and ESSs. Section 5 illustrates the examples of the hunting game before a conclusion is presented.

\section{A simple model}

Before introducing the hunting game, we analyze the strike example that is a simple model of playing the field. This simple game provides an insight on how an individual's payoff is jointly determined by their own strategy and the frequency distribution of other individuals' strategies.

Consider a multi-player game in which the workers decide to start a strike for more pay. The benefit after the strike has positive correlation with the 
75 population of participants and the price they paid. However, the workers can also select non-participation in which they do not need to pay the price but obtain the same benefit as the participants. Let $C$ and $D$ denote the strategy of participation and non-participation respectively. The payoff matrix of the strike example is similar to that of the public goods game $[22,23,28]$ and detailed in

so Tab. 1, where $\{c: c>0\}$ is the cost each worker paid for the strike, $\{k: k>0\}$ is the enhancement factor denoting the production efficiency or synergy effects of cooperation, $\{n: 0 \leq n \leq N\}$ is the number of workers using participation strategy and $\{N: N>2\}$ is the group size of workers.

\begin{tabular}{ll} 
Table 1: Payoff matrix of the strike example \\
Strategy & Payoff \\
\hline$C$ & $E_{C}=\frac{k n c}{N}-c \quad(n \neq 0)$ \\
$D$ & $E_{D}=\frac{k n c}{N} \quad(n \neq N)$ \\
\hline
\end{tabular}

It is easy to see that the individual's payoff is jointly determined by their own strategy and the frequency of other strategies within the population. Note that the payoff of strategy $D$ is always non-negative.

It is possible to determine the number of workers required to choose strategy $C$ so that all strategy $C$ players receive a positive payoff. The solution can be obtained as

$$
E_{c}=\frac{k n c}{N}-c>0
$$

then we have

$$
n>\frac{N}{k} .
$$

Since $0 \leq n \leq N$, we also note that

$$
k>1 .
$$

It is also possible to locate the stable point in this game and its corresponding constraint conditions. First assume that the pure strategy $C$ is a stable point and all workers choose strategy $C$, then the payoff of each individual is

$$
E_{C}=(k-1) c .
$$


If there is only one worker choosing strategy $D$, and the others choose strategy $C$, then we have

$$
\begin{aligned}
& E_{C}=\frac{k(N-1) c}{N}-c \\
& E_{D}=\frac{k(N-1) c}{N} .
\end{aligned}
$$

The pure strategy $C$ is a stable point if it can resist invasion from a mutation, i.e. the following condition is met

$$
(k-1) c>\frac{k(N-1) c}{N}
$$

from which we have

$$
k>N \text {. }
$$

By assuming that the pure strategy $D$ is a stable point, where all workers choose strategy $D$, the payoff for each individual is zero. If there is only one worker choosing strategy $C$ and the others choosing strategy $D$, then we have

$$
\begin{aligned}
E_{C} & =\frac{k c}{N}-c \\
E_{D} & =\frac{k c}{N}
\end{aligned}
$$

Therefore, the pure strategy $D$ is a stable point if the following condition is met

$$
\frac{k c}{N}-c<0
$$

from which we have

$$
k<N
$$

Moreover, there is no mix strategy that can be a stable point because of $E_{C}<E_{D}$ for any $0<n<N$.

In this simple model, either a pure strategy $C$ or $D$ can produce a stable state, with an ESS if $k>N$ or $k<N$ respectively, where there is no stable mix strategy. The strike example is a special case of playing the field, as it is not always true that choosing cooperation can obtain the maximum return. In most cases, the maximum payoff is not clear as the players cannot predict at which point the maximum return can be obtained. In Section 3, a similar but more complex and general model of playing the field is given. 


\section{A general model of playing the field}

Consider games in which there are two subtypes or morphs of one species with two strategies. The strategy using team work to achieve a goal is regarded as a cooperation strategy, denoted by $C$, while the strategy that the individual

100

105

achieves a goal independently is regarded as a defection strategy, denoted by $D$. The payoff of each strategy is jointly determined by its own strategy and the frequency distribution of other individuals' strategies. Let $x$ represent the frequency of strategy $C$ in the population, then the frequency of strategy $D$ is $1-x$. Let $E_{C}(x)$ and $E_{D}(x)$ denote the payoff of an individual playing $C$ and $D$ respectively when the frequency of strategy $C$ is given by $x$. The payoff matrix of the general model for playing the field is give in Tab. 2.

Table 2: Payoff matrix of the general model for playing the field

\begin{tabular}{ll} 
Strategy & Payoff \\
\hline$C$ & $E_{C}(x)$ \\
$D$ & $E_{D}(x)$ \\
\hline
\end{tabular}

Most models of evolutionary biology use "fitness" to assess a specific strategy in the population $[2,3,32]$. The fitness of strategy $C, D$ and the average fitness of the population $(\phi)$ are given by

$$
\begin{aligned}
& f_{C}(x)=f_{0}+x E_{C}(x) \\
& f_{D}(x)=f_{0}+(1-x) E_{D}(x) \\
& \phi=x f_{C}(x)+(1-x) f_{D}(x)
\end{aligned}
$$

where $\left\{f_{0}: f_{0}>0\right\}$ is the baseline fitness of the individual with the same values for all group members, which represents the basic benefit for the population from the environment. For a single, infinite, population of players, the standard model of evolutionary selection dynamics is the replicator equations $[1,10,12]$. The basic idea of the replicator equations is that the more fit a strategy is, at any moment, the more likely it is to be adopted in the future. This can be 
regarded as a diffusion mechanism where individuals tend to switch to strategies that are doing better, or where the fitter individuals have a greater number of offspring [10]. The growth of each strategy is given by the difference between its fitness and the average fitness of the population

$$
x^{\prime}=x\left(f_{C}-\phi\right) .
$$

Since $\phi=x f_{C}+(1-x) f_{D}$, then Eq. 13 can be written as

$$
x^{\prime}=x(1-x)\left(f_{C}-f_{D}\right)
$$

where $f_{0}$ has no effect on this replicator equation. Another replicator equation proposed by [10] can provide similar evolutionary selection dynamics

$$
x(t+1)=x(t) \frac{f_{C}}{\phi} .
$$

where $f_{0}$ can be a control parameter to adjust the convergence speed of the evolutionary process.

The dynamics of the frequency distribution of the two strategies represents the evolutionary game dynamics. The system goes to a stable state when the frequency distribution of the two strategies no longer change.

\section{The hunting game}

We now use the general model of playing the field to investigate a hunting game, in which an individual in a group has to choose either the group hunter. The model of the hunting game may vary according to the payoff of each strategy. We introduce two models of the hunting game: $\alpha$ hunting game and $\beta$ hunting game. 


\section{1. $\alpha$ hunting game}

Consider a hunting game with only two pure strategies: the group hunting strategy $C$ and the individual hunting strategy $D$. The payoff of using the group hunting strategy $C$ increases with the population and the frequency of the strategy but has an upper limit $b$. The price of using the group hunting strategy $C$ decreases with the population and the frequency of the strategy. The payoff of using the individual hunting strategy $D$ is independent of the population and the frequency of the strategy. Suppose that the individuals select the group hunting strategy $C$ with strategy frequency $x$, the payoff matrix of the $\alpha$ hunting game is give in Tab. 3, where $\{N: N>2\}$ is the group size, $\{c: c>0\}$ is the cost coefficient, $\{b: b>0\}$ is the maximum utility when an individual plays strategy $C$, and $\left\{b_{0}: b_{0}>0\right\}$ is a constant that represents the utility when an individual plays strategy $D$. For practical purpose, we have $c>b>b_{0}>0$.

Table 3: Payoff matrix of the $\alpha$ hunting game

\begin{tabular}{ll} 
Strategy & Payoff \\
\hline$C$ & $b-\frac{c}{x N+1}$ \\
$D$ & $b_{0}$ \\
\hline
\end{tabular}

In the $\alpha$ hunting game, the payoff of an individual using the group hunting strategy $C$ is dependent on the population size and the frequency of strategy selection. Fig. 1 displays the variation for a population of 100 where the frequency of strategy $C$ selection is varied.

The fitness of the strategy $C, D$ and the average fitness $(\phi)$ of the population are given by

$$
\begin{aligned}
& f_{C}(x)=f_{0}+x\left(b-\frac{c}{x N+1}\right) \\
& f_{D}(x)=f_{0}+(1-x) b_{0} \\
& \phi=x f_{C}(x)+(1-x) f_{D}(x) .
\end{aligned}
$$

Let $f_{0}=N$ and take Eq. (15) as the replicator equation, then the selection dynamics can be formulated as a Moran process with frequency-dependent fit- 


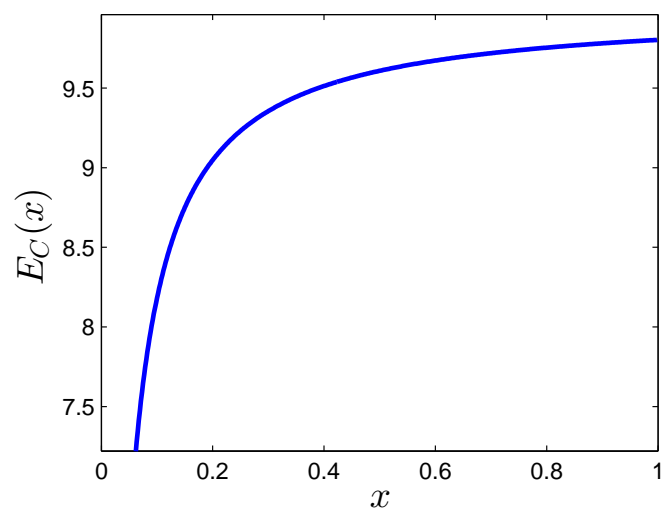

Figure 1: The payoff of the strategy $C$ in the $\alpha$ hunting game. $N=100, b=10, c=20$.

ness $[33,34,35]$. At each time step, replace each individual with offspring whose fitness are dependant on their predecessor. Thus $N$ is strictly constant [36].

Let $f(x)=f_{C}(x)-f_{D}(x)$, then we have the fitness difference equation

$$
f(x)=\left(N b+N b_{0}\right) x^{2}+\left(b-c-N b_{0}+b_{0}\right) x-b_{0}=0 .
$$

The fitness difference function is a parabola function. The coefficients of the parabola function can be represented as

$$
\begin{aligned}
& k_{1}=N b+N b_{0} \\
& k_{2}=b-c-N b_{0}+b 0 \\
& k_{3}=-b_{0} .
\end{aligned}
$$

Then Eq. (17) can be simplified as

$$
f(x)=k_{1} x^{2}+k_{2} x+k_{3}=0 .
$$

For $c>b>b_{0}>0$ and $N>2$, the fitness difference function has the following features

$$
\begin{aligned}
& k_{1}=N b+N b_{0}>0 \\
& \Delta=k_{2}^{2}-4 k_{1} k_{3}=\left(b-c-N b_{0}+b_{0}\right)^{2}+4 b_{0}\left(N b+N b_{0}\right)>0 \\
& f(0)=-b_{0}<0 .
\end{aligned}
$$


With the features of the fitness difference function, the graph of the fitness difference function falls into two types according to the values of $f(1)$, as shown in Fig. 2.

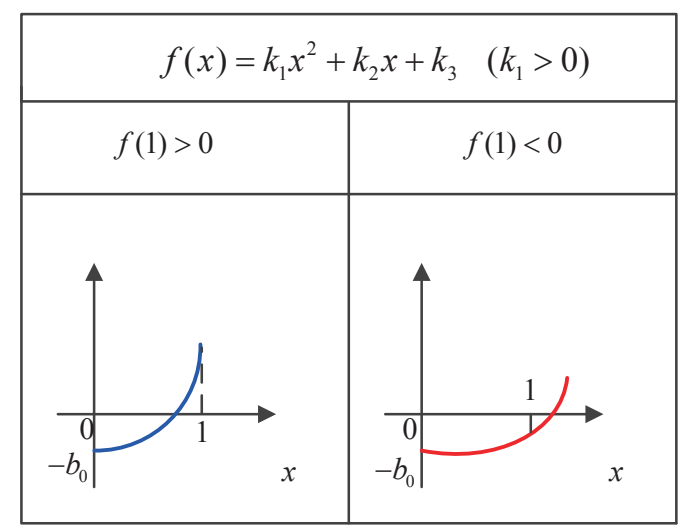

Figure 2: Two graphs of the fitness difference function in the $\alpha$ hunting game

Let $\left\{\left(x_{1}, x_{2}\right): x_{1}<x_{2}\right\}$ be the solutions of the fitness difference equation (Eq. (17)), then for $f(1)>0$, i.e. $N>\frac{c-b}{b}$, we have

$$
\begin{aligned}
& f\left(x_{2}\right)=0 \\
& f^{\prime}\left(x_{2}\right)>0 \\
& 0<x_{2}=\frac{-k_{2}+\sqrt{k_{2}^{2}-4 k_{1} k_{3}}}{2 k_{1}}<1 .
\end{aligned}
$$

The frequency-dependent strategy selection in this case is shown in Fig. 3. 150 The red curve denotes the fitness difference function $f(x)=f_{C}(x)-f_{D}(x)$. If $f(x)>0$, the frequency of strategy $C$ increases; If $f(x)<0$, the frequency of strategy $C$ decreases, the blue arrows indicate the selection direction; If $f(x)=0$, the frequency of strategy $C$ no longer changes, the selection dynamics converges to an equilibrium state. In this case, $x=x_{2}$ is an unstable equilibrium point, and there are two evolutionarily stable strategies (ESSs), the pure group hunting strategy $C$ and the pure individual hunting strategy $D$. The initial frequency distribution of the two strategies determines which specific strategy can be the ESS of the system. If $x_{0}<x_{2}\left(x_{0}\right.$ is the initial frequency of the strategy $\left.C\right)$, 
then the pure individual hunting strategy $(x=0)$ is the ESS with the evolutionary process; If $x_{0}>x_{2}$, then the pure group hunting strategy $(x=1)$ is the ESS with the evolutionary process; A mixed hunting strategy $x=x_{2}$ cannot be the ESS because it cannot resist invasion from a mutation.

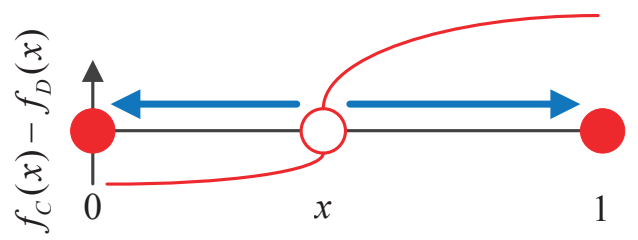

Figure 3: The frequency-dependent strategy selection in the $\alpha$ hunting game when $f(1)>0$. - denotes an ESS, o denotes an unstable equilibrium point, $\rightarrow$ denotes the selection direction and $\frown$ presents the fitness difference function.

For $f(1)<0$, i.e. $N<\frac{c-b}{b}$, we have

$$
\begin{aligned}
& f\left(x_{2}\right)=0 \\
& f^{\prime}\left(x_{2}\right)>0 \\
& x_{2}>1 \\
& f(x)<0 \quad x \in[0,1] .
\end{aligned}
$$

The frequency-dependent strategy selection in this case is shown in Fig. 4. The fitness difference function is negative for any $0 \leq x \leq 1$, making the pure individual hunting strategy D the only ESS.

For $f(1)=0$, i.e. $N=\frac{c-b}{b}$, then $x=1$ is an unstable equilibrium point and it can be only obtained by $x_{0}=1$.

In fact $f(1)>0$ means that all individuals choosing the group hunting strategy $C$ can obtain a greater payoff than that of any mutational individual choosing the individual hunting strategy $D$. This scenario leads to the results of frequency-dependent strategy selection, as indicated by Fig. 3. While $f(1)<0$ means that all individuals choosing the group hunting strategy $C$ cannot obtain as much payoff as an individual choosing the individual hunting strategy $D$. 


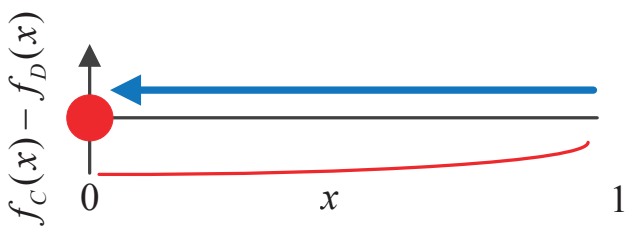

Figure 4: The frequency-dependent strategy selection in the $\alpha$ hunting game when $f(1)<0$. - denotes an ESS, o denotes an unstable equilibrium point, $\rightarrow$ denotes the selection direction and $\frown$ presents the fitness difference function.

Hence, the individual hunting strategy $D$ becomes the only ESS as indicated by Fig. 4.

The selection dynamics also shows that the stable states are independent of $b_{0}$, which means that a constant payoff of the individual hunting strategy $C$ with $c>b>b_{0}$ has no effect on the ESS in the $\alpha$ hunting game. Another interesting result is that the worst time to play the group hunting strategy $C$ is at the point

$$
x=-\frac{k_{2}}{2 k_{1}}
$$

because the fitness difference reaches a minimum.

\section{2. $\beta$ hunting game}

Consider a more complicated hunting game with only two pure strategies: the group hunting strategy $C$ and the individual hunting strategy $D$. For the group hunting strategy $C$, suppose that a few cooperators cannot obtain a positive utility in group hunting until there are enough participants. After reaching this threshold, the payoff increases with the population, but it reverses to negative when there are too many participants. The payoff of the individual hunting strategy $D$ is independent of the frequency and the population. Let $x$ be the frequency of individuals selecting the group hunting strategy $C$, then the payoff matrix of the $\beta$ hunting game is give in Tab. 4 , where $0<h_{1}<h_{2}<1$, $a<0, b_{0}>0$ and $N>2$. 
Table 4: Payoff matrix of the $\beta$ hunting game

\begin{tabular}{ll} 
Strategy & Payoff \\
\hline$C$ & $\frac{a}{N^{2}}\left(x+\frac{1}{N}-h_{1}\right)\left(x+\frac{1}{N}-h_{2}\right)$ \\
$D$ & $b_{0}$ \\
\hline
\end{tabular}

The payoff of the group hunting strategy $C$ has a general form of parabola function, as shown in Fig. 5.

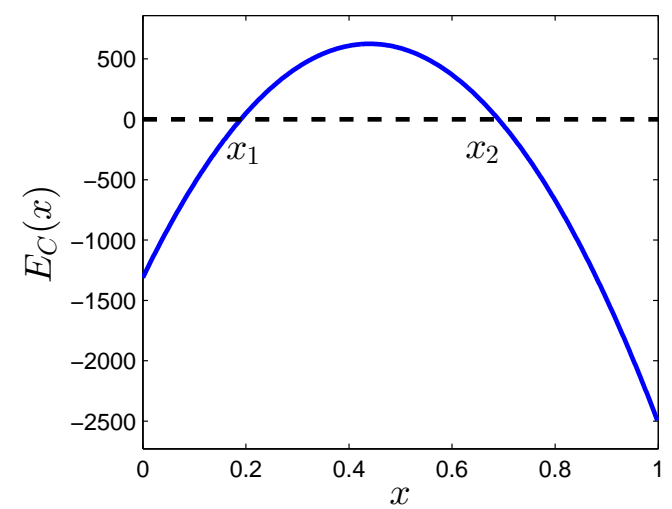

Figure 5: The payoff of the strategy $C$ in the $\beta$ hunting game. $N=100, b=10, c=20$, $b_{0}=5$.

The fitness of the strategy $C, D$ and the average fitness $(\phi)$ of the population are given by

$$
\begin{aligned}
& f_{C}(x)=f_{0}+x\left(\frac{a}{N^{2}}\left(x+\frac{1}{N}-h_{1}\right)\left(x+\frac{1}{N}-h_{2}\right)\right) \\
& f_{D}(x)=f_{0}+(1-x) b_{0} \\
& \phi=x f_{C}(x)+(1-x) f_{D}(x) .
\end{aligned}
$$

Without loss of generality, let $f_{0}=N^{2}$, take Eq. (15) to be the replicator equation. Let $f(x)=f_{C}(x)-f_{D}(x)$, then we have the fitness difference function

$$
\begin{aligned}
f(x)= & a N^{2} x^{3}+\left(2 a N-a h_{1} N^{2}-a h_{2} N^{2}\right) x^{2} \\
& +\left(a-a h_{1} N-a h_{2} N+a h_{1} h_{2} N^{2}+b_{0}\right) x-b_{0} .
\end{aligned}
$$


Let

$$
\begin{aligned}
& k_{1}=a N^{2} \\
& k_{2}=2 a N-a h_{1} N^{2}-a h_{2} N^{2} \\
& k_{3}=a-a h_{1} N-a h_{2} N+a h_{1} h_{2} N^{2}+b_{0} \\
& k_{4}=-b_{0} .
\end{aligned}
$$

Therefore, the fitness difference equation in the $\beta$ hunting game can be given by the standard form of the cubic equation.

$$
f(x)=k_{1} x^{3}+k_{2} x^{2}+k_{3} x+k_{4}=0 .
$$

The discriminants of the cubic equation is given by [37] as

$$
\begin{aligned}
& A=k_{2}^{2}-3 k_{1} k_{3} \\
& B=k_{2} k_{3}-9 k_{1} k_{4} \\
& C=k_{3}-3 k_{2} k_{4} \\
& \Delta=B^{2}-4 A C .
\end{aligned}
$$
in the $\beta$ hunting game falls into two types, as shown in Fig. 6.

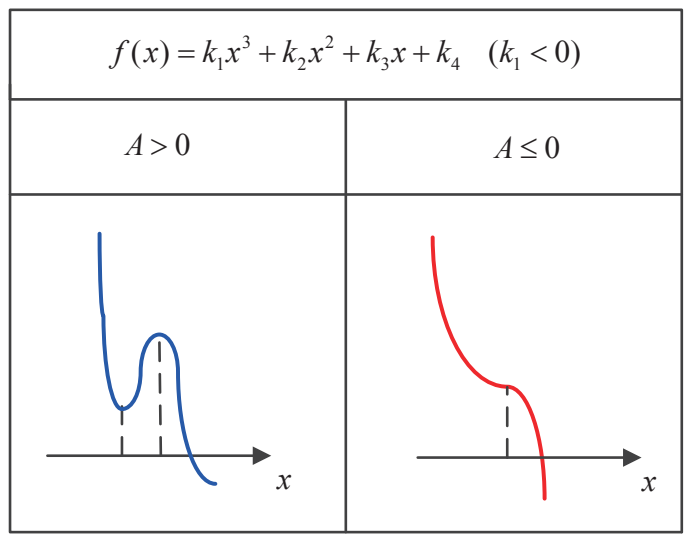

Figure 6: Two graphs of the fitness difference function in the $\beta$ hunting game according to the discriminants. 
Let $x_{1}, x_{2}$ and $x_{3}$ be the solutions of the fitness difference equation (Eq. (27)).

(1) If $\Delta=B^{2}-4 A C>0$, the fitness difference equation has a single real solution

$$
x_{1}=x_{2}=x_{3}=\frac{-k_{2}-\left(\sqrt[3]{Y_{1}}+\sqrt[3]{Y_{2}}\right)}{3 k_{1}}
$$

where $Y_{1,2}=A k_{2}+3 k_{1}\left(\frac{-B \pm \sqrt{B^{2}-4 A C}}{2}\right)$.

For both $A>0$ and $A \leq 0$, Fig. 6 demonstrates $f^{\prime}\left(x_{1,2,3}\right)<0$ if Eq. (27) has a single real solution (i.e., $f(x)>0$ when $x<x_{1,2,3} ; f(x)<0$ when $x>x_{1,2,3}$ ). According to the range of $x_{1,2,3}$, there are three frequency-dependent strategy selection scenarios. Fig. 7 displays that $x=x_{1,2,3}$ is a stable equilibrium point, as well as an ESS if $0<x_{1,2,3}<1$, because both selection direction arrows point at this equilibrium point. Moreover, either $x=0$ or $x=1$ is a stable equilibrium point (ESS) if $x_{1,2,3}<0$ or $x_{1,2,3}>1$ respectively.

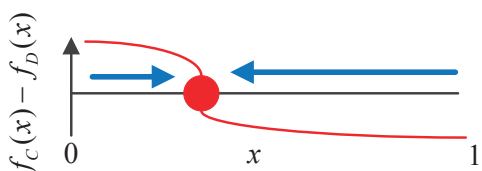

(a) $0<x_{1,2,3}<1$

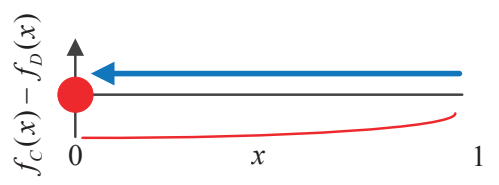

(b) $x_{1,2,3}<0$

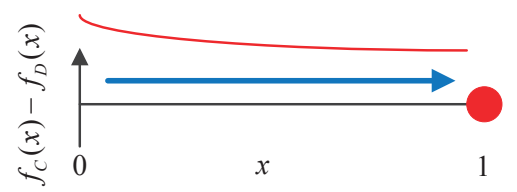

(c) $x_{1,2,3}>1$

Figure 7: The frequency-dependent strategy selection in the $\beta$ hunting game with $\Delta>0$. $\bullet$ denotes an ESS, o denotes an unstable equilibrium point, $\rightarrow$ denotes the selection direction and $\frown$ presents the fitness difference function.

(2) If $\Delta=B^{2}-4 A C=0$, the fitness difference equation has two different real solutions

$$
\begin{aligned}
& x_{1}=\frac{-k_{2}}{k-1}+M \\
& x_{2}=x_{3}=\frac{-M}{2}
\end{aligned}
$$


where $M=\frac{B}{A},(A \neq 0)$.

As we can see from Fig. 6, the fitness difference function has two different real solutions only when $A>0$. The solutions have two different distributions: $x_{1}<x_{2}=x_{3}$ and $x_{2}=x_{3}<x_{1}$. For $x_{1}<x_{2}=x_{3}$, there are six frequencydependent strategy selection scenarios, see Fig. 8. For $x_{2}=x_{3}<x_{1}$, there are another six frequency-dependent strategy selection scenarios, see Fig. 9.

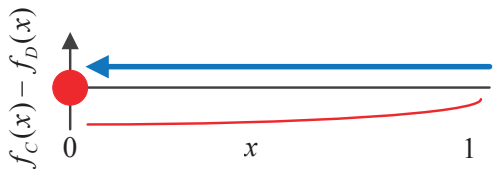

(a) $x_{1}<x_{2}=x_{3}<0$

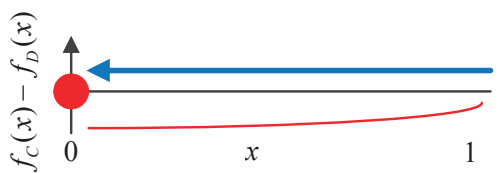

(c) $x_{1}<0<1<x_{2}=x_{3}$

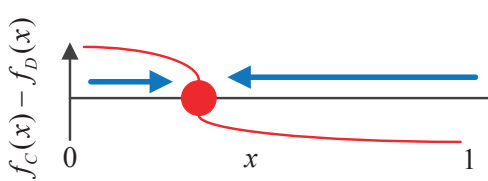

(e) $0<x_{1}<1<x_{2}=x_{3}$

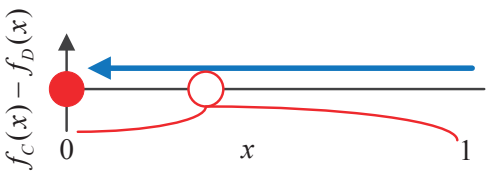

(b) $x_{1}<0<x_{2}=x_{3}<1$

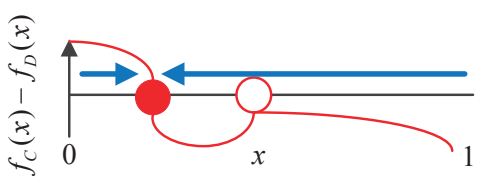

(d) $0<x_{1}<x_{2}=x_{3}<1$

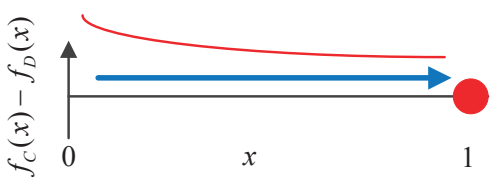

(f) $1<x_{1}<x_{2}=x_{3}$

Figure 8: The frequency-dependent strategy selection in the $\beta$ hunting game with $\Delta=0$ and $x_{1}<x_{2}=x_{3}$. $\bullet$ denotes an ESS, $\circ$ denotes an unstable equilibrium point, $\rightarrow$ denotes the selection direction and $\frown$ presents the fitness difference function.

Note that, the system can have two equilibrium points as indicated in Fig. 8(b), Fig. 8(d), Fig. 9(d) and Fig. 9(e). These results show that an equilibrium point is unstable if the fitness function maintains the sign at both sides of the equilibrium point.

(3) If $\Delta=B^{2}-4 A C<0$, the fitness difference equation has three different 


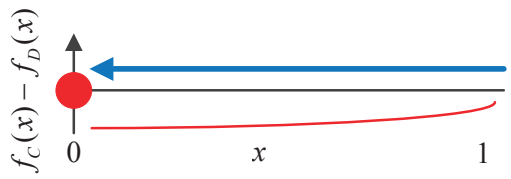

(a) $x_{2}=x_{3}<x_{1}<0$

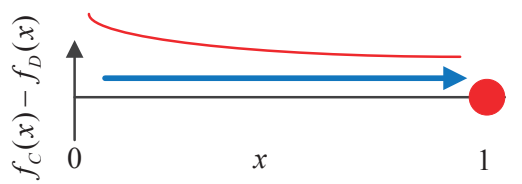

(c) $x_{2}=x_{3}<0<1<x_{1}$

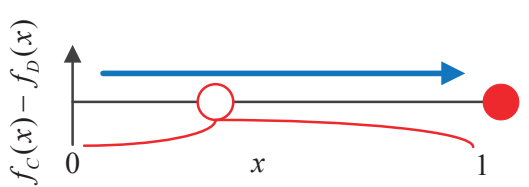

(e) $0<x_{2}=x_{3}<1<x_{1}$

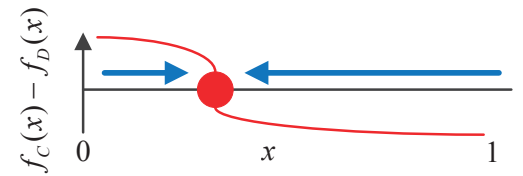

(b) $x_{2}=x_{3}<0<x_{1}<1$

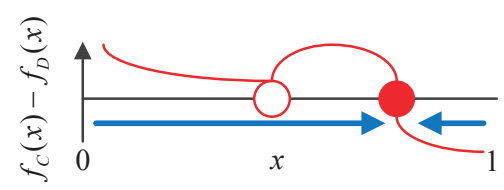

(d) $0<x_{2}=x_{3}<x_{1}<1$

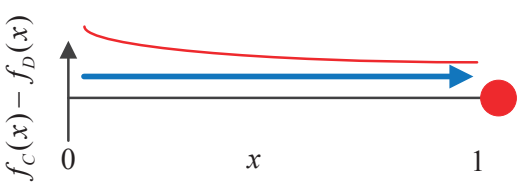

(f) $1<x_{2}=x_{3}<x_{1}$

Figure 9: The frequency-dependent strategy selection in the $\beta$ hunting game with $\Delta=0$ and $x_{2}=x_{3}<x_{1}$. $\bullet$ denotes an ESS, ○ denotes an unstable equilibrium point, $\rightarrow$ denotes the selection direction and $\frown$ presents the fitness difference function.

real solutions

$$
\begin{aligned}
& x_{1}=\frac{-k_{2}-2 \sqrt{A} \cos \frac{\theta}{3}}{3 k_{1}} \\
& x_{2,3}=\frac{-k_{2}+\sqrt{A}\left(\cos \frac{\theta}{3} \pm \sqrt{3} \sin \frac{\theta}{3}\right)}{3 k_{1}}
\end{aligned}
$$

where

$$
\begin{aligned}
& \theta=\arccos (T) \\
& T=\frac{2 A k_{2}-3 k_{1} B}{\left.2 \sqrt{(} A^{3}\right)}
\end{aligned}
$$

and $A>0,-1<T<1$.

There are eight frequency-dependent strategy selection scenarios in this case according to the ranges of the three solutions, see Fig. 10.

Fig. 10 displays that the system may have one, two or three equilibrium points which can be either stable or unstable equilibrium points. The pure 


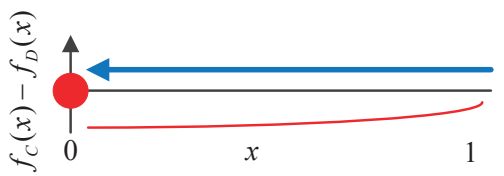

(a) $x_{1}<x_{2}<x_{3}<0$

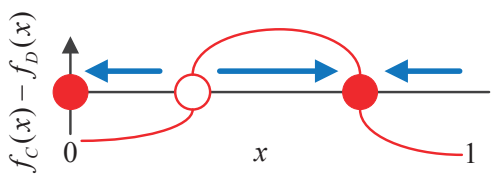

(c) $x_{1}<0<x_{2}<x_{3}<1$

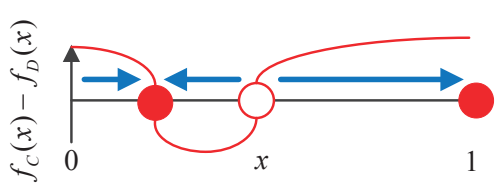

(e) $0<x_{1}<x_{2}<1<x_{3}$

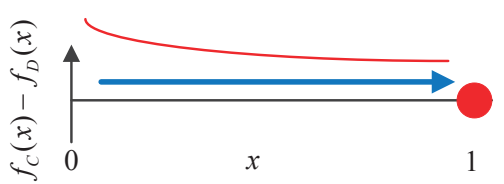

(g) $1<x_{1}<x_{2}<x_{3}$

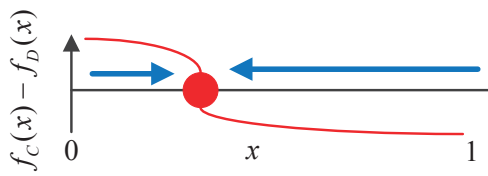

(b) $x_{1}<x_{2}<0<x_{3}<1$

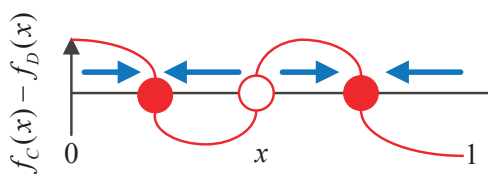

(d) $0<x_{1}<x_{2}<x_{3}<1$

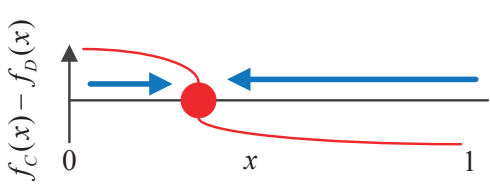

(f) $0<x_{1}<1<x_{2}<x_{3}$

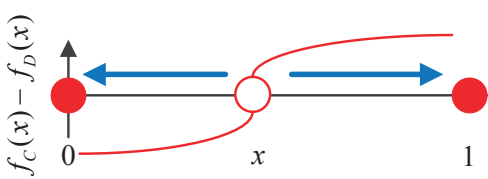

(h) $x_{1}<0<x_{2}<1<x_{3}$

Figure 10: The frequency-dependent strategy selection in the $\beta$ hunting game with $\Delta<0$. • denotes an ESS, ○ denotes an unstable equilibrium point, $\rightarrow$ denotes the selection direction and $\frown$ presents the fitness difference function.

strategies $(x=0$ or $x=1)$ are always equilibrium points where $x=0$ is a stable equilibrium point if $f(0)<0$ and $x=1$ is a stable equilibrium point if $f(1)>0$. An internal equilibrium point $\left(0<x_{1,2,3}<1\right.$ and $\left.f\left(x_{1,2,3}=0\right)\right)$ is a stable equilibrium point if $f^{\prime}\left(x_{1,2,3}\right)<0$; It is an unstable equilibrium point if $f^{\prime}\left(x_{1,2,3}\right)>0$. For example, when $0<x_{1}<x_{2}<x_{3}<1$, the system has three equilibrium points, where $x_{1}$ and $x_{3}$ are stable equilibrium points, and $x_{2}$ is an unstable equilibrium point, as indicated in Fig. 10(d).

The next section is to present how to obtain the stable/unstable equilibrium points and ESSs in the hunting game. 
Given a general playing the field model with two pure strategies, we can always get the fitness difference equation, with which equilibrium points can be solved. Let $x$ be the frequency of one strategy, an internal equilibrium point $x$ is a stable equilibrium point if

$$
\left\{\begin{array}{r}
0<x<1 \\
f(x)=0 \\
f^{\prime}(x)<0
\end{array}\right.
$$

It is an unstable equilibrium point if

$$
\left\{\begin{array}{r}
0<x<1 \\
f(x)=0 \\
f^{\prime}(x) \geq 0
\end{array}\right.
$$

The concept of ESS was introduced by $[2,3]$ to describe the long-run effects of selection for more successful strategies where the ESS resists invasion from a mutation. This idea motivates the following definition:

Definition 1. A state $x$ is called an ESS (evolutionarily stable strategy) in the 230 hunting game if for every state $y \neq x$, if let $y=x+\varepsilon$ and let $y=x-\varepsilon$ (the perturbed state), then $f_{C}(y)<f_{D}(y)$ and $f_{C}(y)>f_{D}(y)$ respectively for sufficiently small $\varepsilon>0$.

The Definition 1 presents that if a state $x$ is an ESS, then both strategy $C$ player and strategy $D$ player have no motivation to shift their strategies.

Take $f(x)=f_{C}(x)-f_{D}(x)$, the Definition 1 can be simplified as follows:

Definition 2. A state $x$ is called an ESS in the hunting game if the following condition for sufficiently small $\varepsilon>0$ is met:

$$
x= \begin{cases}x: & 0<x<1, \quad f(x)=0, \quad f^{\prime}(x)<0 \\ 0: & f(0+\varepsilon)<0 \\ 1: & f(1-\varepsilon)>0 .\end{cases}
$$


The Definition 2 presents that a stable equilibrium point will alwyas act as an ESS, while an unstable equilibrium point cannot act as an ESS.

\section{Examples}

The conclusions of stable/unstable equilibrium points, ESSs and the selection dynamics in the $\alpha$ and $\beta$ hunting games are verified in this section through numerical simulations.

\section{1. $\alpha$ hunting game}

Let $N=100, f_{0}=N, b=10, c=20$, thus $N=100>\frac{c-b}{b}=1$. As we can see from Fig. 11, $x=x_{2}$ is an unstable equilibrium point. If $x_{0}<x_{2}$, the $\alpha$ hunting game evolves into the pure individual hunting strategy $D(x=0)$; If $x_{0}>x_{2}$, the $\alpha$ hunting game evolves into the pure group hunting strategy $C$ $(x=1)$. The frequency-dependent strategy selection is consistent with Fig. 3 because of $N>\frac{c-b}{b}$.

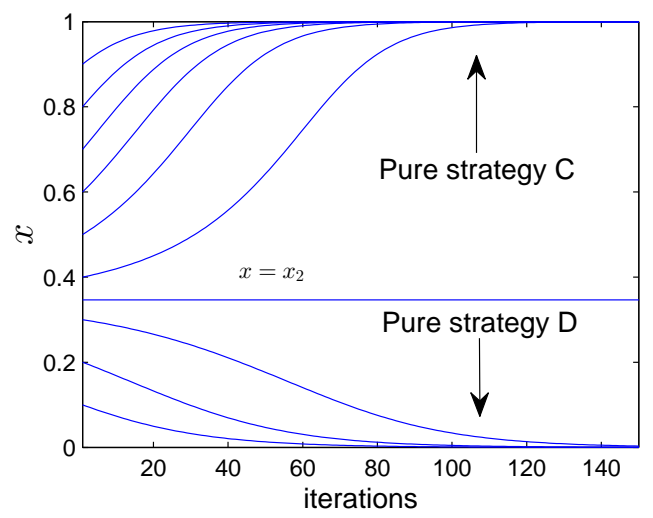

Figure 11: The evolutionary history of strategy frequency in the $\alpha$ hunting game with $N>$ $\frac{c-b}{b}$.

Here we have

$$
x_{2}=\frac{-k_{2}+\sqrt{k_{2}^{2}-4 k_{1} k_{3}}}{2 k_{1}}=0.3463 .
$$


Let $N=100, f_{0}=N, b=1, c=200$, thus $N=100<\frac{c-b}{b}=199$. Fig. 12 shows that the $\alpha$ hunting game evolves into the pure individual hunting strategy $D(x=0)$ only. The frequency-dependent strategy selection is consistent with Fig. 4 because of $N<\frac{c-b}{b}$.

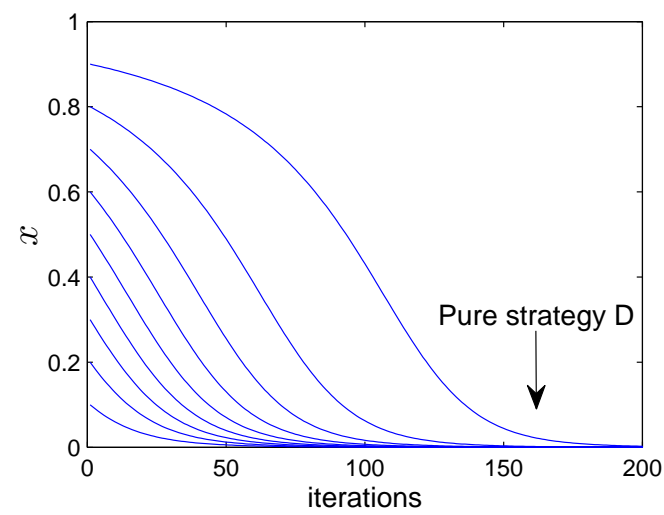

Figure 12: The evolutionary history of strategy frequency in the $\alpha$ hunting game with $N<$ $\frac{c-b}{b}$.

Since $b_{0}$ describes the payoff of an individual using the individual hunting strategy $D$, a larger value of $b_{0}$ can speed up the evolutionary strategy $D$ selection, but slow down the evolutionary strategy $C$ selection. However, it has no effect on the final stable states as we mentioned in Section 4.1. $f_{0}$ is not a critical parameter to the evolutionary dynamics, especially when Eq. (14) is used as the replicator equation. It just affects the convergence speed of ESS when Eq. (15) is used as the replicator equation. This is not always negative to the evolutionary game. For example, by introducing and adjusting the parameter $f_{0}$, the diffusion speed of one strategy among the population can be controlled. These conclusions about $f_{0}$ are also shown to be valid for the $\beta$ hunting game, in the following section.

\section{2. $\beta$ hunting game}

Let $N=100, f_{0}=N^{2}, a=-1, b=10, c=20, b_{0}=5, h_{1}=0.2, h_{2}=0.7$. The specific fitness difference function of the $\beta$ hunting game is shown in Fig. 
13. In this case, we have

$$
\Delta=B^{2}-4 A C=-1.3 \times 10^{14}<0 .
$$

According to Eq. (31), the $\beta$ hunting game has three different real solutions and $x_{1}<0<x_{2}<x_{3}<1$, as indicated in Fig. 13. This is one of eight selection scenarios when $\Delta<0$, as shown in Fig. 10(c). Consistent with the previous analysis in Section 4.3, $x_{2}$ is an unstable equilibrium point while $x_{3}$ is a stable equilibrium point. We have

$$
\begin{aligned}
& x_{2}=\frac{-k_{2}+\sqrt{A}\left(\cos \frac{\theta}{3}-\sqrt{3} \sin \frac{\theta}{3}\right)}{3 k_{1}}=0.1942 \\
& x_{3}=\frac{-k_{2}-2 \sqrt{A} \cos \frac{\theta}{3}}{3 k_{1}}=0.6895 .
\end{aligned}
$$

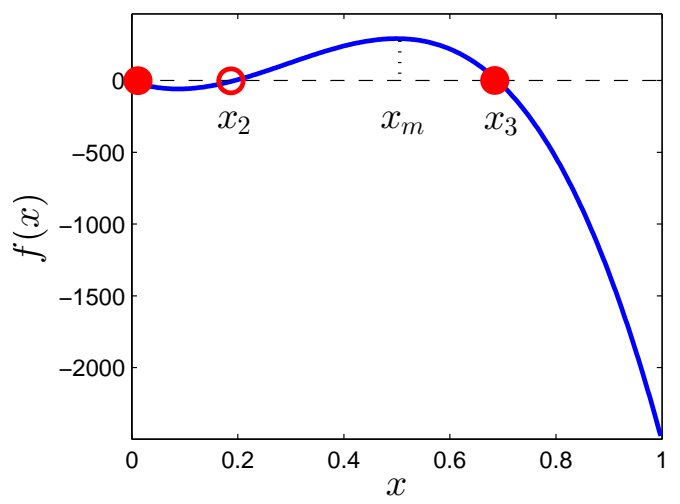

Figure 13: The fitness difference function in the $\beta$ hunting game

265 hunting game. The results show that if $x_{0}<x_{2}$, the $\beta$ hunting game evolves into the pure individual hunting strategy $D$. If $x_{2}<x_{0}<x_{3}$ or $x_{0}>x_{3}$, the $\beta$ hunting game evolves into the mixed strategies, where the stable frequency of the strategy $C$ is $x=x_{3} . x=x_{2}$ is an unstable equilibrium point, which is to 270 appear only when $x_{0}=x_{2}$. 


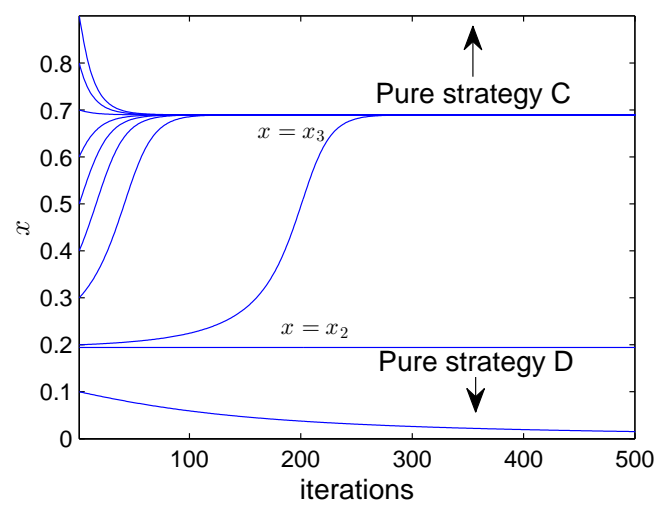

Figure 14: The evolutionary history of strategy frequency in the $\beta$ hunting game with $\Delta<0$.

\subsection{Conflict between frequency-dependent selection and population fitness}

From the perspective of population interests, it is best to obtain the optimum state when the system evolves into the evolutionarily stable state. The evolutionary mechanism given by Eq. (14) and Eq. (15) can make the hunting game evolve into evolutionarily stable states. However, the stable states are not always the optimum states for the whole group in terms of population fitness. For example, in the $\alpha$ hunting game, the stable state $x=0$ is not beneficial to the whole group, while $x=1$ is a stable state that can lead to the best population fitness. In the $\beta$ hunting game, both stable states $x=0$ and $x=x_{3}$ are not optimal to the whole group with regards to population fitness. A case of fitness evolution in the $\beta$ hunting game is shown in Fig. 15. It indicates that the best frequency distribution of strategies, in terms of population fitness, is obtained before the system reaches the stable state.

The stable strategies emerging in the frequency-dependent strategy selection, using Eq. (14) and Eq. (15), are not always the optimal strategies for the whole group. This is mainly because the diffusion mechanism of one strategy is implemented by considering the fitness difference between strategies. The population fitness may be indirectly considered from the individuals' standpoint, which is out of the scope of this paper. 


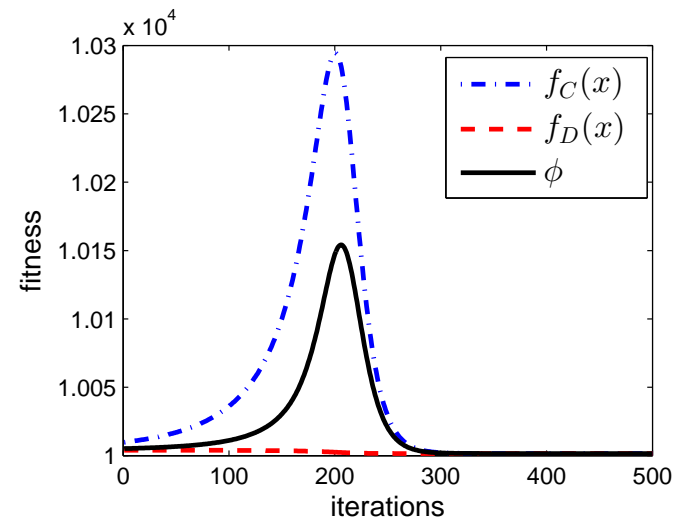

Figure 15: A case of fitness evolution in the $\beta$ hunting game with $x_{0}=0.2, N=100, f_{0}=$ $N^{2}, a=-1, b=10, c=20, b_{0}=5, h_{1}=0.2, h_{2}=0.7$.

\section{Conclusion}

This paper proposed a new game termed the hunting game in the playing the field model, where there are two subtypes of one species with two strategies: the group hunting strategy and the individual hunting strategy. The evolutionary dynamics of this hunting game are investigated by considering the Moran process. Various selection scenarios are considered where each generates different stable and unstable equilibrium points.

The evolutionarily stable strategies (ESSs) for each hunting game are studied by using the deterministic replicator equations and fitness difference functions. The results from the hunting games reveal that choosing a strategy that has a higher benefit, in the playing the field model at each iteration, will always lead to an evolutionarily stable state.

The evolutionarily stable state is found to be not always unique because the system can have multiple equilibrium points. It is shown that a stable equilibrium point will always act as an ESS, while an unstable equilibrium point cannot act as an ESS because the system cannot resist invasion from a mutation. It is seen that if the slope of the fitness difference function at the equilibrium point is negative, the equilibrium point will act as a stable equilibrium point. 
The pure strategies $(x=0$ or $x=1$ ) are always equilibrium points where $x=0$ is a stable equilibrium point if $f(0+\varepsilon)<0$ and $x=1$ is a stable equilibrium point if $f(1-\varepsilon)>0$.

The results also present that an individual cannot predict when its payoff has reached an optimum. Moreover, the population fitness cannot always reach an optimum level when applying the evolutionary process with the fitness difference function, and the ESS is not favoured by group selection, which was also found by $[2,32,38]$.

The hunting game in the playing the field model is generally existed. It is practically significant to understand the frequency-dependent strategy selection as well as the evolutionarily stable strategies in this multi-player game. With stable/unstable equilibrium points and ESSs, one can predict the selection dynamics of an extended playing the field system as long as the better strategy is allowed to be diffused in the group.

Future work will investigate how to implicitly guide as few individuals as possible, so that they choose a strategy that optimizes the population fitness as the system evolves.

\section{Acknowledgements}

This work was partially supported by the National Natural Science Foundation of China (NSFC) under Grants 51379176, 51679201.

\section{References}

[1] J. Hofbauer, K. Sigmund, Evolutionary games and population dynamics, Cambridge university press, 1998.

[2] J. M. Smith, The theory of games and the evolution of animal conflicts, Journal of theoretical biology 47 (1) (1974) 209-221.

[3] J. M. Smith, Evolution and the Theory of Games, Cambridge university press, 1982. 
[4] J. Gore, H. Youk, A. Van Oudenaarden, Snowdrift game dynamics and facultative cheating in yeast, Nature 459 (7244) (2009) 253.

[5] A. Rapoport, A. M. Chammah, The game of chicken, American Behavioral Scientist 10 (3) (1966) 10-28.

[6] R. Axelrod, W. D. Hamilton, The evolution of cooperation, science 211 (4489) (1981) 1390-1396.

[7] W. Deng, K. Huang, C. Yang, H. Zhu, Z. Yu, Promote of cooperation in networked multiagent system based on fitness control, Applied Mathematics and Computation 339 (2018) 805-811.

[8] A. Bandyopadhyay, S. Kar, Coevolution of cooperation and network structure in social dilemmas in evolutionary dynamic complex network, Applied Mathematics and Computation 320 (2018) 710-730.

[9] C. Liu, J. Shi, T. Li, J. Liu, Aspiration driven coevolution resolves social dilemmas in networks, Applied Mathematics and Computation 342 (2019) $247-254$.

[10] P. D. Taylor, L. B. Jonker, Evolutionary stable strategies and game dynamics, Mathematical biosciences 40 (1-2) (1978) 145-156.

[11] S. J. Schreiber, Urn models, replicator processes, and random genetic drift, SIAM Journal on Applied Mathematics 61 (6) (2001) 2148-2167.

[12] J. Hofbauer, K. Sigmund, Evolutionary game dynamics, Bulletin of the American Mathematical Society 40 (4) (2003) 479-519.

[13] M. Benaim, S. J. Schreiber, P. Tarres, et al., Generalized urn models of evolutionary processes, The Annals of Applied Probability 14 (3) (2004) 1455-1478.

[14] M. Shpak, S. H. Orzack, E. Barany, The influence of demographic stochasticity on evolutionary dynamics and stability, Theoretical population biology 88 (2013) 47-56. 
[15] Z. Ding, S. Wang, H. Yang, Evolutionarily stable strategy and invader strategy in matrix games, Journal of mathematical biology 66 (1-2) (2013) 383-397.

[16] D. A. Charlebois, G. Balázsi, Frequency-dependent selection: a diversifying force in microbial populations, Molecular systems biology 12 (8) (2016) 880.

[17] O. Aydogmus, Discovering the effect of nonlocal payoff calculation on the stabilty of ess: Spatial patterns of hawk-dove game in metapopulations, Journal of theoretical biology 442 (2018) 87-97.

[18] C. L. Hall, M. A. Porter, M. S. Dawkins, Dominance, sharing, and assessment in an iterated hawk-dove game, Journal of theoretical biology (2019) 110101.

[19] G. Bornstein, Intergroup conflict: Individual, group, and collective interests, Personality and social psychology review 7 (2) (2003) 129-145.

[20] V. P. Crawford, On the definition of an evolutionarily stable strategy in the playing the field model, Journal of theoretical Biology 143 (2) (1990) 269-273.

[21] T. Platkowski, J. Stachowska-Pietka, Esss in n-player mixed games, Applied mathematics and computation 167 (1) (2005) 592-606.

[22] F. C. Santos, M. D. Santos, J. M. Pacheco, Social diversity promotes the emergence of cooperation in public goods games, Nature 454 (7201) (2008) 213.

[23] R.-R. Liu, C.-X. Jia, Z. Rong, Effects of enhancement level on evolutionary public goods game with payoff aspirations, Applied Mathematics and Computation 350 (2019) 242-248.

[24] G. Hardin, The tragedy of the commons, science 162 (3859) (1968) 12431248. 
[25] M.-h. Chen, L. Wang, S.-w. Sun, J. Wang, C.-y. Xia, Evolution of cooperation in the spatial public goods game with adaptive reputation assortment, Physics Letters A 380 (1-2) (2016) 40-47.

[26] J. Du, Redistribution promotes cooperation in spatial public goods games under aspiration dynamics, Applied Mathematics and Computation 363 (2019) 124629.

[27] B. Gao, S. Hou, D. Jia, M. Du, et al., Resolving public goods dilemma by giving the poor more support, Applied Mathematics and Computation 362 (2019) 124529.

[28] J. Quan, Y. Zhou, X. Wang, J.-B. Yang, Information fusion based on reputation and payoff promotes cooperation in spatial public goods game, Applied Mathematics and Computation 368 (2020) 124805.

[29] N. Chung, J. Pienaar, J. M. Greeff, Evolutionary stable sex ratios with non-facultative male-eggs first sex allocation in fig wasps, Oikos 128 (6) (2019) 859-868.

[30] M. E. Chudzińska, F. M. van Beest, J. Madsen, J. Nabe-Nielsen, Using habitat selection theories to predict the spatiotemporal distribution of migratory birds during stopover-a case study of pink-footed geese anser brachyrhynchus, Oikos 124 (7) (2015) 851-860.

[31] T. Lux, M. Marchesi, Scaling and criticality in a stochastic multi-agent model of a financial market, Nature 397 (6719) (1999) 498.

[32] M. A. Nowak, Five rules for the evolution of cooperation, science 314 (5805) (2006) 1560-1563.

[33] J. C. Claussen, A. Traulsen, Non-gaussian fluctuations arising from finite populations: Exact results for the evolutionary moran process, Physical review E 71 (2) (2005) 025101. 
[34] C. Taylor, Y. Iwasa, M. A. Nowak, A symmetry of fixation times in evoultionary dynamics, Journal of theoretical biology 243 (2) (2006) 245-251.

[35] W. Huang, A. Traulsen, Fixation probabilities of random mutants under frequency dependent selection, Journal of theoretical biology 263 (2) (2010) 262-268.

[36] M. A. Nowak, A. Sasaki, C. Taylor, D. Fudenberg, Emergence of cooperation and evolutionary stability in finite populations, Nature 428 (6983) (2004) 646.

[37] S. Fan, A new extracting formula and a new distinguishing means on the one variable cubic equation, Nat. Sci. J. Hainan Teach. Coll 2 (2) (1989) 91-98.

${ }_{425}$ [38] M. A. Nowak, Evolutionary dynamics, Harvard University Press, 2006. 